\title{
Leukemic Diffuse Large B-Cell Lymphoma in a Patient With Myeloproliferative Disorder
}

\author{
Vijaya Raj Bhatt, MBBS ; R. Gregory Bociek, MDa; Ji Yuan, $\mathrm{PhD}^{\mathrm{b}}$; Kai Fu, MD, PhD ${ }^{\mathrm{b}}$; Timothy C. Greiner, $\mathrm{MD}^{\mathrm{b}}$; \\ Bhavana J. Dave, $\mathrm{PhD}^{c}$; Sandeep K. Rajan, $\mathrm{MD}^{\mathrm{a}}$; and James O. Armitage, MD
}

\begin{abstract}
Essential thrombocythemia is well-known to transform to other myeloid disorders, such as leukemia; however, the risk for development of lymphoma is not as well studied. This case report discusses a 76-year-old man with a history of prefibrotic post-essential thrombocythemia myelofibrosis on ruxolitinib, who developed anemia, thrombocytopenia, and leukocytosis with peripheral blasts. Results of a bone marrow biopsy and PET and CT scans revealed stage IV leukemic diffuse large B-cell lymphoma. Several days after cessation of ruxolitinib, the patient developed fevers, hypotension, and low-grade disseminated intravascular coagulation, and subsequently developed spontaneous tumor lysis syndrome, which resulted in death. This case is unique in several aspects: it highlights the rare possibility of lymphomatous transformation of myeloproliferative disorders, an unusual presentation of lymphoma masquerading as leukemia, and the possibility of ruxolitinib withdrawal syndrome. Additionally, this case serves as a reminder that the use of novel therapies should be adopted after a thorough assessment of long-term risks, including those associated with abrupt withdrawal. (J Natl Compr Canc Netw 2015;13:281-287)
\end{abstract}

\section{NCCN: Continuing Education}

\section{Accreditation Statement}

This activity has been designated to meet the educational needs of physicians and nurses involved in the management of patients with cancer. There is no fee for this article. No commercial support was received for this article. The National Comprehensive Cancer Network (NCCN) is accredited by the ACCME to provide continuing medical education for physicians.

\footnotetext{
From the a Department of Internal Medicine, Division of HematologyOncology; 'Department of Pathology and Microbiology; and cHuman Genetics Laboratory, Munroe Meyer Institute for Genetics and Rehabilitation, University of Nebraska Medical Center, Omaha, Nebraska. Submitted February 15, 2014; accepted for publication June 6, 2014. Dr. Rajan had disclosed that he is a consultant for Baxter International Inc., Alexion Pharmaceuticals, Biogen Idec, and Incyte Corporation; he receives honoraria from Alexion Pharmaceuticals; is on the speakers' bureau for Alexion Pharmaceuticals and Biogen Idec; and receives research support from Incyte Corporation. Dr. Armitage has disclosed that he is a consultant for Celgene Corporation, GlaxoSmithKline, Roche, Spectrum Pharmaceuticals, Inc., and ZIOPHARM Oncology, Inc; and is on the Board of Directors for TESARO, Inc. The remaining authors have disclosed that they have no financial interests, arrangements, affiliations, or commercial interests with the manufacturers of any products discussed in this article or their competitors.

Correspondence: Vijaya Raj Bhatt, MBBS, University of Nebraska Medical Center, Department of Internal Medicine, Division of HematologyOncology, 987680 Nebraska Medical Center, Omaha, NE 68198-7680. E-mail: vijaya.bhatt@unmc.edu
}

NCCN designates this journal-based CME activity for a maximum of 1.0 AMA PRA Category 1 Credit(s) ${ }^{\mathrm{TM}}$. Physicians should claim only the credit commensurate with the extent of their participation in the activity.

NCCN is accredited as a provider of continuing nursing education by the American Nurses Credentialing Center's Commission on Accreditation.

This activity is accredited for 1.0 contact hours. Accreditation as a provider refers to recognition of educational activities only; accredited status does not imply endorsement by NCCN or ANCC of any commercial products discussed/displayed in conjunction with the educational activity. Kristina M. Gregory, RN, MSN, OCN, is our nurse planner for this educational activity.

All clinicians completing this activity will be issued a certificate of participation. To participate in this journal CE activity: 1) review the learning objectives and author disclosures; 2 ) study the education content; 3) take the posttest with a $66 \%$ minimum passing score and complete the evaluation at http://education.nccn.org/ node/62686; and 4) view/print certificate.

Release date: March 6, 2015; Expiration date: March 6, 2016

\section{Learning Objectives}

Upon completion of this activity, participants will be able to:

- Summarize the use of ruxolitinib and its potential side effects in patients with post-essential thrombocythemia myelofibrosis

- Identify the various mechanisms related to the transformation of essential thrombocythemia into NHL

\section{EDITOR}

Kerrin M. Green, MA, Assistant Managing Editor, JNCCN—Journal of the National Comprehensive Cancer Network

Ms. Green has disclosed that she has no relevant financial relationships.

\section{CE AUTHORS}

Deborah J. Moonan, RN, BSN, Director, Continuing Education, has disclosed that she has no relevant financial relationships.

Ann Gianola, MA, Manager, Continuing Education Accreditation \& Program Operations, has disclosed that she has no relevant financial relationships.

Kristina M. Gregory, RN, MSN, OCN, Vice President, Clinical Information Operations, has disclosed that she has no relevant financial relationships. Rashmi Kumar, PhD, Senior Manager, Clinical Content, has disclosed that she has no relevant financial relationships.

Hema Sundar, PhD, Oncology Scientist/Senior Medical Writer, has disclosed that she has no relevant financial relationships. 
Overall survival in essential thrombocythemia is excellent, with some patients living as long as the general population. ${ }^{1}$ Over time, essential thrombocythemia may develop into other myeloid disorders, such as post-essential thrombocythemia myelofibrosis or acute leukemia, with less than a $5 \%$ risk of leukemic transformation in essential thrombocythemia, which is considered the lowest among myeloproliferative neoplasms. ${ }^{2}$ However, distinction from early or prefibrotic primary myelofibrosis is important because the latter is associated with a greater risk of progression to leukemic transformation and death. ${ }^{1}$ In essential thrombocythemia, the risk of leukemic transformation increases with advanced age, anemia, platelet count greater than $1000 \times 10^{9} / \mathrm{L},{ }^{2}$ and after the first decade from diagnosis. ${ }^{3}$ Additionally, the development of myelofibrosis can increase the risk for subsequent leukemic transformation. ${ }^{4}$ Although leukemic transformation is a well-known complication of essential thrombocythemia, the risk for development of lymphoma is not as well studied.

\section{Case Report}

A 76-year-old man was referred to the University of Nebraska Medical Center (UNMC) for multiple episodes of epistaxis; skin bruises; intermittent fevers; fatigue; anorexia; a few episodes of diarrhea; and dyspnea during the preceding week. At the referring institution, he was found to have severe anemia and thrombocytopenia, and received platelet and packed red blood cell transfusions. A nasal endoscopy was performed and a bleeding vessel was cauterized.

Previous medical history was significant for chronic kidney disease (CKD) stage III, hypertension, hypertriglyceridemia, gout, benign prostatic hypertrophy, transurethral resection of prostate, laser resection of penile cancer, resection of squamous cell carcinoma of the skin, and essential thrombocythemia with $\operatorname{del}(9)(q 13 q 22)$. Essential thrombocythemia, diagnosed 12 years previously, was initially treated with hydroxyurea until 10 months before presentation, when it was discontinued because of the development of leg ulcers, and anagrelide was initiated. Seven months before presentation, he developed weight loss, intermittent fever, symptomatic splenomegaly with the tip palpable $6 \mathrm{~cm}$ below the left costal margin, new anemia (hemoglobin level, $10.4 \mathrm{~g} / \mathrm{dL}$ ), and elevated lactate dehydrogenase (LDH) levels of 407
$\mathrm{U} / \mathrm{L}$ (normal $\leq 220 \mathrm{U} / \mathrm{L}$ ). Quantitative reverse transcription-polymerase chain reaction was negative for BCR/ABL1. Results of a bone marrow biopsy showed nearly $100 \%$ cellularity, with 3\% blasts and numerous atypical megakaryocytes of mononuclear and hypolobulated bizarre forms and areas of megakaryocyte aggregates. Reticulin staining showed grade 1 to 3 reticulin fibrosis. JAK2 V617F mutation was positive. Early post-essential thrombocythemia myelofibrosis was diagnosed, and ruxolitinib treatment was initiated and the dose of anagrelide reduced, which resulted in improvement of weight loss and fever and the resolution of splenomegaly. One month before presentation to the referring institution, the patient developed progressive anemia with a hemoglobin level of $8.3 \mathrm{~g} / \mathrm{dL}$, which was attributed to CKD and ruxolitinib use, and he was started on epoetin alfa. Other home medications included vitamin D, furosemide, gemfibrozil, hydrochlorothiazide, losartan, metoprolol, tamsulosin, aliskiren, aspirin, ruxolitinib, and anagrelide. Ruxolitinib, anagrelide, and aspirin were withheld by the referring institution 1 week before transfer to UNMC. Family history was significant for lymphosarcoma in his father and colon cancer in a daughter at the age of 25 years.

On physical examination at UNMC, the patient had blood pressure of $162 / 98 \mathrm{~mm} \mathrm{Hg}$, heart rate of 90 beats per minute, temperature of $37.8^{\circ} \mathrm{C}$, and respiratory rate of 20 breaths per minute. He had multiple ecchymoses over both upper and lower extremities, enlargement of multiple cervical and inguinal lymph nodes, and dried blood in the posterior pharynx and nasal cavities. His abdomen was distended, but soft and nontender. The spleen was palpable $8 \mathrm{~cm}$ below the left costal margin. The remainder of the examination was unremarkable.

Laboratory evaluation revealed WBC count of $12,000 / \mathrm{mcL}$ with $48 \%$ blasts, hemoglobin level of $11.3 \mathrm{~g} / \mathrm{dL}$, platelet count of $24,000 / \mathrm{mcL}$, prothrombin time of 17.7 seconds, activated partial thromboplastin time of 30 seconds, fibrinogen level of 454 $\mathrm{mg} / \mathrm{dL}, \mathrm{D}$-dimer value of greater than $4.0 \mathrm{mcg} / \mathrm{mL}$, and antithrombin activity of $57 \%$ (suggestive of low-grade disseminated intravascular coagulation). Peripheral blood showed numerous circulating lymphoma cells but no schistocytes (Figure 1). Serum creatinine level was $2.3 \mathrm{mg} / \mathrm{dL}$, blood urea nitrogen level was $98 \mathrm{mg} / \mathrm{dL}$, and levels of sodium and potassium were 131.0 and $3.7 \mathrm{mmol} / \mathrm{L}$, respectively. Results 


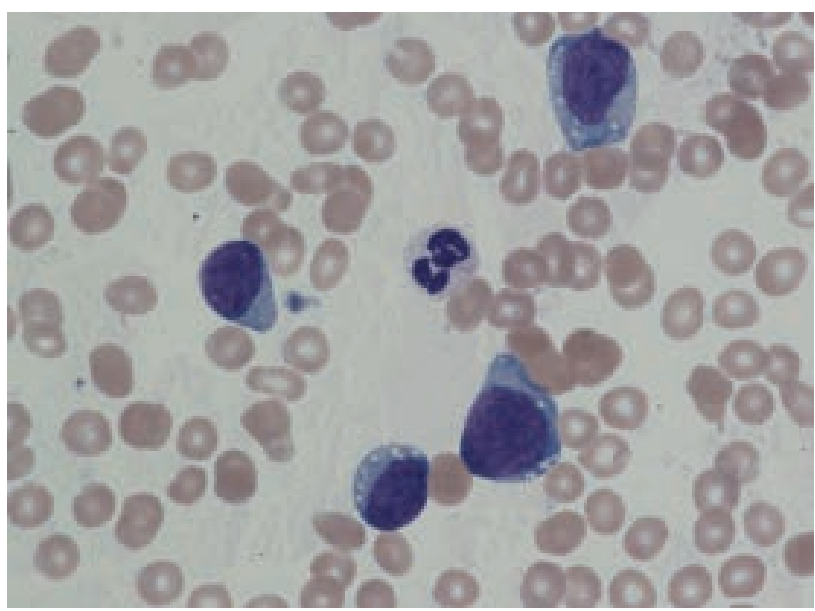

Figure 1 Peripheral blood smear shows several large cells with irregular nuclear contour, open chromatin, small nucleoli, deep-blue cytoplasm, and cytoplasmic vacuoles (Wright-Giemsa, original magnification $\times 1000$ ).

for other blood chemicals, including bicarbonate and calcium, and liver-function tests were unremarkable. Urine and blood cultures were negative.

Based on these findings, the patient was started on allopurinol and intravenous normal saline infusion. Two days after hospitalization, his temperature increased to $38.2^{\circ} \mathrm{C}$ and he was started on intravenous cefepime. Results of repeat urine and blood cultures were negative. Bone marrow biopsy showed markedly hypercellular bone marrow (95\%) with increased large lymphoid cells, decreased myeloid and erythroid cells, increased megakaryocytes with abnormal morphology and dysplasia (Figure 2), and grade $3 / 3$ reticulin fibrosis (Figure 3 ). Flow cytometry of the bone marrow showed a population of intermediate to large-sized mature B cells expressing CD19, CD20, CD38, CD45, BCL6, MUM1, and moderatedensity $\lambda$ light chains (Figure 4 ), but lacked CD3, CD5, CD10, CD33, and CD34, and were negative for myeloperoxidase or Sudan Black B. Cytogenetic analysis revealed a complex karyotype with more than 5 cytogenetic aberrations, including $\operatorname{del}(17)$ (p11.2p13). Fluorescence in situ hybridization studies showed rearrangement of BCL6 but not BCL2 or CMYC (see Appendix 1, available online, in this article, at JNCCN.org). Integrated ${ }^{18} \mathrm{~F}$-fluorodeoxyglucose PET/CT scan of the skull to mid-thigh showed multiple enlarged and hypermetabolic cervical, mediastinal, retrocrural, iliac, and inguinal lymph nodes; an enlarged spleen at $20 \mathrm{~cm}$ with a maximum standardized uptake value of 7.2; and a small splenic infarct (Figure 5). Therefore, a diagnosis of stage IV

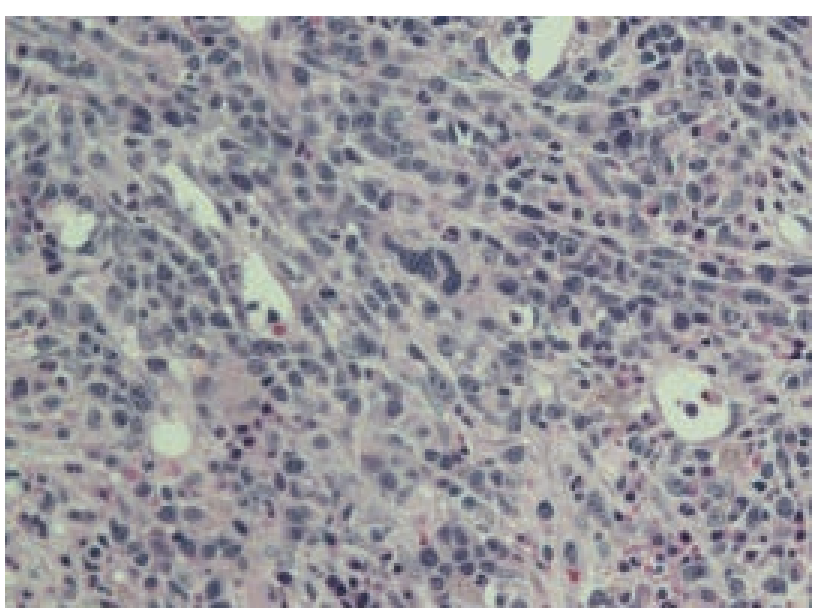

Figure 2 Bone marrow biopsy shows marked hypercellularity with sheets of large lymphoid cells containing multiple small nucleoli (Hematoxylin and eosin, original magnification $\times 400$ ).

leukemic diffuse large B-cell lymphoma (DLBCL), activated B-cell subtype was established.

After a thorough discussion, the patient and his family decided against future resuscitation and intubation. Subsequently, the patient developed hypotension that was managed with fluid resuscitation and discontinuation of antihypertensive medications. He did not develop any further fevers and was otherwise stable. On day 5 of hospitalization, the patients' absolute lymphoma cell count decreased from 5600 to $500 / \mathrm{mcL}$. Laboratory test results showed increased levels of creatinine $(4.4 \mathrm{mg} / \mathrm{dL})$, blood urea nitrogen $(127 \mathrm{mg} / \mathrm{dL}), \mathrm{LDH}$ (767 U/L; normal up to $192 \mathrm{U} / \mathrm{L})$, uric acid (20.9 mg/dL), phosphorus $(7.4 \mathrm{mg} / \mathrm{dL})$, metabolic acidosis (bicarbonate of $16 \mathrm{mmol} / \mathrm{L}$ and anion gap of $17 \mathrm{mmol} / \mathrm{L})$, potassium $(4.7 \mathrm{mmol} / \mathrm{L})$, and calcium $(8.6 \mathrm{~g} / \mathrm{dL})$. A diagnosis of spontaneous tumor lysis syndrome (TLS) with acute kidney injury in addition to CKD was established. Rasburicase was administered, and the patient received 1 session of hemodialysis. Soon after, the patient became increasingly oliguric and developed a metabolic encephalopathy. At this point, the family decided to switch to comfort measures only, and the patient died peacefully on day 6 of hospitalization.

\section{Discussion}

The patient developed DLBCL 12 years after diagnosis of myeloproliferative disorder. Although essential thrombocythemia is known to transform into 


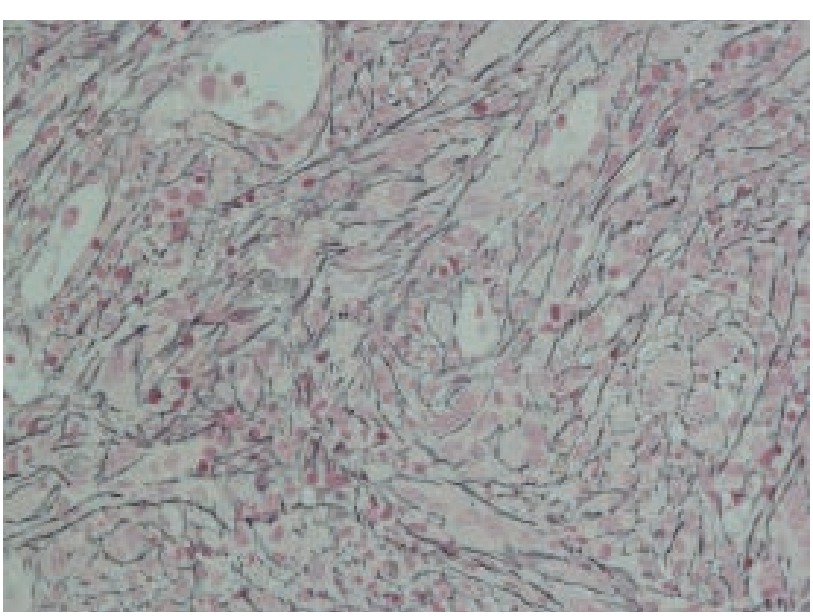

Figure 3 Reticulin stain on bone marrow biopsy shows grade 3 of 3 reticulin fibrosis (reticulin, original magnification $\mathrm{x} 400$ ).

other myeloid disorders, such as acute leukemia, this case illustrates the rare possibility of developing lymphoma and an unusual presentation of DLBCL with circulating lymphoma cells. From 1996 to 2010, only 29 cases of leukemic DLBCL were diagnosed at UNMC and Emory University School of Medicine. ${ }^{5}$ The unusual presentation of lymphoma masquerad- ing as leukemia and the rarity of the association between myeloproliferative disorders and lymphoma can potentially result in a misdiagnosis of acute leukemia. Therefore, a high index of suspicion and a thorough bone marrow biopsy evaluation using immunohistochemistry, flow cytometry, and cytogenetic studies and traditional morphological assessment are important for accurate diagnosis.

Patients with essential thrombocythemia are at an increased risk of developing hematologic malignancies, such as non-Hodgkin's lymphoma (NHL), and nonhematologic malignancies. In a Danish population-based cohort study, the standardized incidence ratios of developing nonhematologic and hematologic malignancies among patients with essential thrombocythemia were 1.2 and 5.0, respectively, compared with the general population. ${ }^{6}$ In an Italian single-center study, standardized incidence ratios for developing NHL and chronic lymphocytic leukemia in patients with essential thrombocythemia were 2.6 and 12.4 , respectively. In 2 of 3 patients with lymphoid neoplasms, the JAK2 V617F mutation was also present in lymphoid cancer cells, thus indicating that the origin

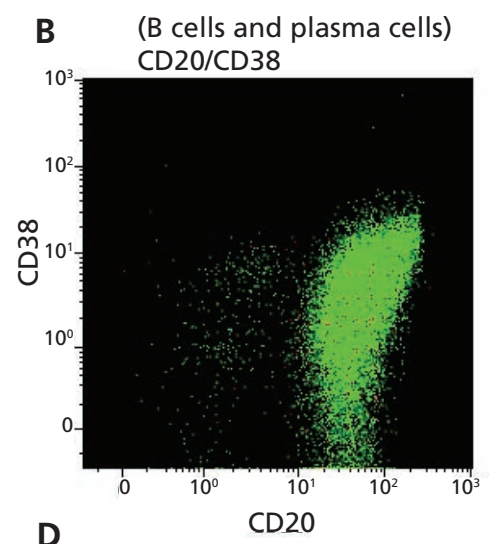

c
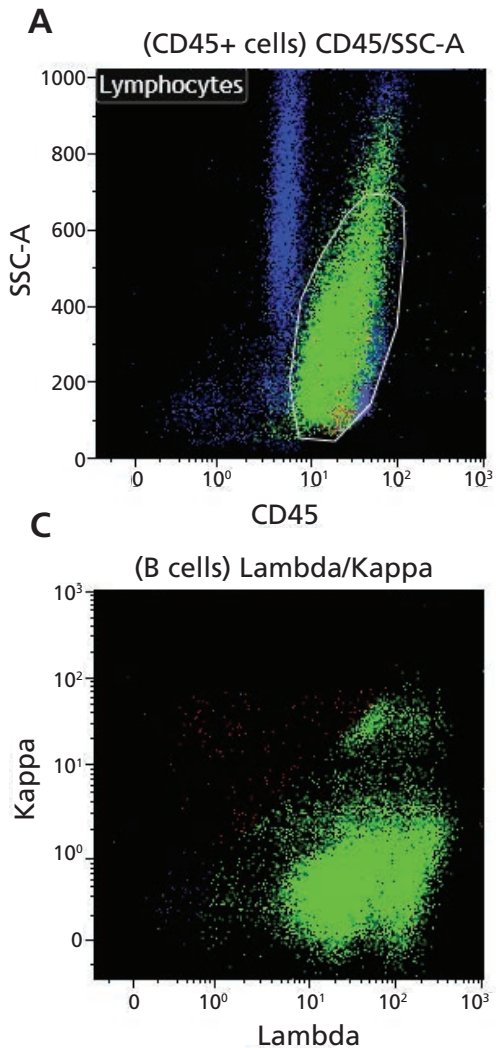

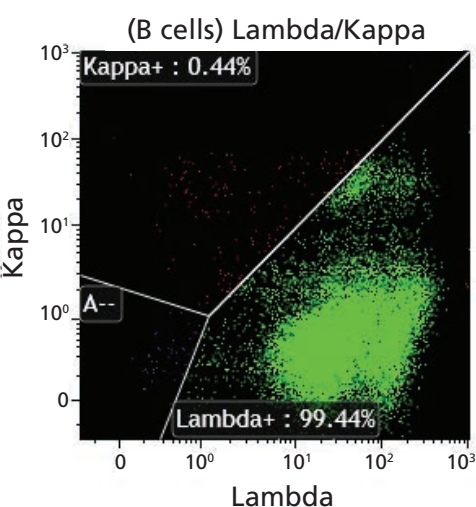

Figure 4 Flow cytometry shows a large population of mature B cells expressing CD45 (A), CD20 and CD38 (B), and moderate-density $\lambda$ light chains ( $C$ and $D)$. 


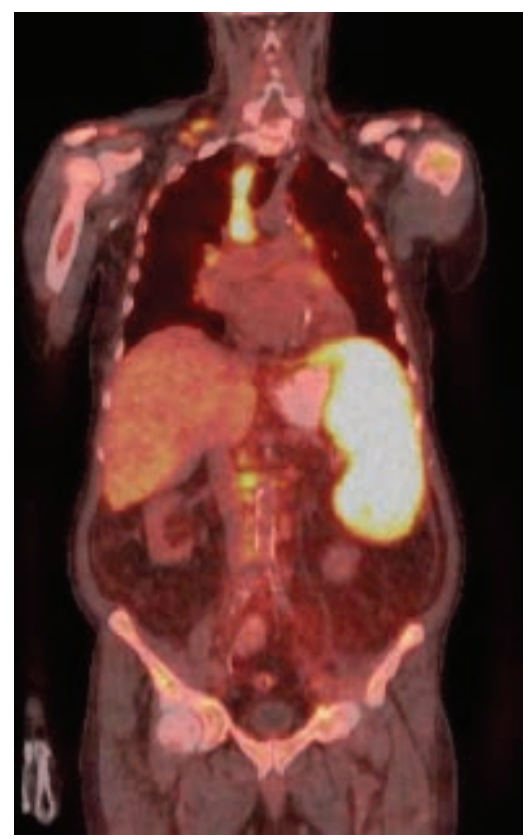

Figure 5 Integrated 18F-fluorodeoxyglucose PET/CT scan shows enlarged and hypermetabolic spleen and multiple lymph nodes.

of cancer was from JAK2 V617F-mutated common lymphoid-myeloid hematopoietic progenitor cells. ${ }^{?}$ JAK2 V617F mutation has been identified in both $\mathrm{B}$ and $\mathrm{T}$ lymphocytes of patients with myeloproliferative neoplasms, ${ }^{8}$ which further supports this possibility. Other postulated mechanisms of development of lymphoma in essential thrombocythemia include the presence of common carcinogen- and therapy-related complications. Autocrine cytokine secretion and activation of JAK are being recognized more in several lymphoma subtypes. In the activated B-cell subtype of DLBCL, as in the present patient, gain-of-function MYD88 mutation results in autocrine secretion of cytokines, such as interleukin (IL)-6 and IL-10, and autocrine JAK activation, which promote survival of cancer cells. In addition, the activation of JAK signaling has been linked with the expression of oncogene MYC in lymphomas. ${ }^{9}$ In primary myelofibrosis, several cytokines, including IL-6 and IL-10, are increased and correlate with specific phenotypic manifestation and prognosis. ${ }^{10}$ Therefore, it is theoretically appealing to hypothesize that JAK activation and an abnormal cytokine profile may be the link between myelofibrosis and DLBCL, activated B-cell subtype. Finally, in one study, $3.5 \%$ of patients with polycythemia vera treated with chlorambucil developed lymphoma, ${ }^{1}$ suggesting the possibility of therapy-related events.
Therapeutic agents can influence the probability of disease transformation in myeloproliferative disorders. Use of alkylating agents, such as chlorambucil and radiophosphorus, have been associated with the development of leukemia, ${ }^{1}$ and therefore have fallen out of favor. Although hydroxyurea has been a concern, it has not been found to be associated with an increased risk of leukemic transformation. ${ }^{1}$ However, the sequential use of more than 1 cytotoxic agent may be a risk factor for leukemic transformation, and hydroxyurea may increase leukemic risk in combination with other cytotoxic agents. ${ }^{11}$ The risk of leukemic transformation with ruxolitinib, which was approved based on 2 phase III trials with a median follow-up of 1 year, is unclear. ${ }^{12,13}$ A subgroup analysis of a phase I/II study with a much longer followup showed an approximately 10\% risk of both leukemic transformation and withdrawal syndrome. ${ }^{14}$ Abrupt cessation of ruxolitinib has been associated with withdrawal syndrome manifested by symptom relapse, painful enlargement of splenomegaly, septic shock-like syndrome, cytopenias, disseminated intravascular coagulation, and tumor lysis. ${ }^{15,16}$ In the present patient with renal dysfunction, ruxolitinib was used in combination with anagrelide. Use of this combination therapy and its use in renal dysfunction are not supported by current evidence. ${ }^{12,13}$ Ruxolitinib should be judiciously used only in symptomatic patients with intermediate- or high-risk myelofibrosis based on the dynamic International Prognostic Scoring System. In the present patient, levels of electrolytes and creatinine were normal at presentation (uric acid and LDH levels were not available); however, within a few days of ruxolitinib cessation, he developed spontaneous TLS and other complications. Therefore, ruxolitinib withdrawal might have contributed to the overall clinical deterioration of the patient. Therapy with ruxolitinib results in significant suppression of inflammatory cytokines, ${ }^{17}$ and its abrupt cessation is thought to result in cytokine rebound resulting in withdrawal syndrome, including TLS. Gradual cessation of ruxolitinib may prevent these complications, whereas the reinitiation of ruxolitinib and use of glucocorticoids may be useful in the management of withdrawal syndrome..$^{15,16}$

The role of various therapeutic agents in the development of lymphomatous transformation in myeloproliferative disorders remains unclear. In one report, a woman with post-polycythemia vera my- 
elofibrosis developed Burkitt-like large B-cell lymphoma with complex karyotype less than 2 months after starting ruxolitinib (prior bone marrow biopsy showed no evidence of lymphoma and cytogenetic abnormalities). ${ }^{15}$ The present patient had DLBCL with complex karyotype and presented with circulating lymphoma cells. Such unusual presentations of common diseases also suggest the possibility of novel or unusual causes, such as ruxolitinib alone or in combination with other agents. Because of the lack of long-term follow-up data on ruxolitinib, further studies are needed to clarify the risk of disease transformation with ruxolitinib and the risk of lymphomatous transformation associated with several therapeutic agents used alone or as a part of sequential therapy in myeloproliferative disorders.

The rarity of lymphomatous transformation and lack of large-scale studies have prevented the development of predictive factors for lymphomatous transformation in myeloproliferative disorder. However, one study showed that the risk of lymphoid malignancies in myeloproliferative neoplasms was greater among men, patients with a JAK2 V617Fpositive mutation, and patients who were 5 years postdiagnosis of myeloproliferative neoplasms. ${ }^{7}$ In one study, ${ }^{3}$ the risk of leukemic transformation increased substantially with time $(1.4 \%, 8.1 \%$, and $24.0 \%$ in the first 10 years, second decade, and third decade, respectively) and contributed to poor survival in those surviving after the first decade. Therefore, it is important to monitor patients for disease transformation 5 to 10 years after diagnosis. In the present patient, in addition to the other factors discussed previously, the personal and familial history of malignancies ${ }^{18}$ and his older age may have played contributory roles in the development of aggressive NHL. ${ }^{19-21}$

A paucity of literature exists regarding the treatment of lymphoma developing concurrently with or after the diagnosis of essential thrombocythemia. In a retrospective study $(\mathrm{N}=8), 4$ patients with lowgrade lymphoma did not require any therapy, whereas 3 patients with stage III-IV follicular lymphoma and 1 patient with DLBCL received lymphoma-type chemotherapy, with all experiencing complete remission of lymphoma and partial or complete remission of essential thrombocythemia. ${ }^{22}$ Thus, it is possible that the presence of essential thrombocythemia does not significantly alter the management or re- mission rate of some patients with lymphoma. However, the present patient and a patient with Burkittlike large B-cell lymphoma that developed soon after ruxolitinib initiation ${ }^{15}$ had complex karyotype and multiple comorbidities, resulting in poor outcomes. Additionally, leukemic DLBCL may have greater early morbidity and mortality. In one study DLBCL masquerading as leukemia was associated with a high tumor burden, frequent extranodal involvement, complex bone marrow cytogenetics, and a high rate of complications and death during induction, but was associated with an approximately 50\% 4-year survival rate in patients able to tolerate anthracycline and rituximab-based regimens. ${ }^{5}$

\section{Conclusions}

This case illustrates that myeloproliferative neoplasms tend to transform to other myeloid disorders, and that patients are also at risk of developing lymphoma. With improvement in survival, it is important to be aware of the natural history of chronic diseases, such as essential thrombocythemia. This understanding allows proper monitoring of potential long-term complications and selection of efficacious yet safer agents from the available therapeutic armamentarium. New therapies are often responsible for improved outcomes; however, their use should be adopted after thorough assessment of their potential efficacy in providing optimal care and the risks associated with long-term therapy, including those related to abrupt withdrawal.

\section{Acknowledgments}

The authors would like to thank Christine Higgins and Pamela Althof for their help in conducting cytogenetic and fluorescence in situ hybridization studies.

\section{References}

1. Tefferi A. Polycythemia vera and essential thrombocythemia: 2012 update on diagnosis, risk stratification, and management. Am J Hematol 2012;87:285-293.

2. Gangat N, Wolanskyj AP, McClure RF, et al. Risk stratification for survival and leukemic transformation in essential thrombocythemia: a single institutional study of 605 patients. Leukemia 2007;21:270-276.

3. Wolanskyj AP, Schwager SM, McClure RF, et al. Essential thrombocythemia beyond the first decade: life expectancy, long-term complication rates, and prognostic factors. Mayo Clin Proc 2006;81:159-166.

4. Chim CS, Kwong YL, Lie AK, et al. Long-term outcome of 231 patients with essential thrombocythemia: prognostic factors for thrombosis, 
Leukemic Diffuse Large B-Cell Lymphoma

bleeding, myelofibrosis, and leukemia. Arch Intern Med 2005;165:26512658.

5. Muringampurath-John D, Jaye DL, Flowers CR, et al. Characteristics and outcomes of diffuse large B-cell lymphoma presenting in leukaemic phase. Br J Haematol 2012;158:608-614.

6. Frederiksen $\mathrm{H}$, Farkas DK, Christiansen CF, etal. Chronic myeloproliferative neoplasms and subsequent cancer risk: a Danish population-based cohort study. Blood 2011;118:6515-6520.

7. Vannucchi AM, Masala G, Antonioli E, et al. Increased risk of lymphoid neoplasms in patients with Philadelphia chromosome-negative myeloproliferative neoplasms. Cancer Epidemiol Biomarkers Prev 2009; 18:2068-2073.

8. Larsen TS, Christensen JH, Hasselbalch HC, Pallisgaard N. The JAK2 V617F mutation involves B-and T-lymphocyte lineages in a subgroup of patients with Philadelphia-chromosome negative chronic myeloproliferative disorders. Br J Haematol 2007;136:745-751.

9. O'Shea JJ, Holland SM, Staudt LM. JAKs and STATs in immunity, immunodeficiency, and cancer. N Engl J Med 2013;368:161-170.

10. Tefferi A, Vaidya R, Caramazza D, et al. Circulating interleukin (IL)-8, IL-2R, IL-12, and IL-15 levels are independently prognostic in primary myelofibrosis: a comprehensive cytokine profiling study. J Clin Oncol 2011;29:1356-1363.

11. Palandri F, Catani L, Testoni N, et al. Long-term follow-up of 386 consecutive patients with essential thrombocythemia: safety of cytoreductive therapy. Am J Hematol 2009;84:215-220.

12. Harrison C, Kiladjian JJ, Al-Ali HK, et al. JAK inhibition with ruxolitinib versus best available therapy for myelofibrosis. N Engl J Med 2012;366:787798.

\section{Instructions for Completion}

To participate in this journal CE activity: 1) review the learning objectives and author disclosures; 2 ) study the education content; 3 ) take the posttest with a $66 \%$ minimum passing score and complete the evaluation at http://education.ncon.org/ node/62686; and 4) view/print certificate. After reading the article, you should be able to answer the following multiple-
13. Verstovsek S, Mesa RA, Gotlib J, et al. A double-blind, placebo-controlled trial of ruxolitinib for myelofibrosis. N Engl J Med 2012;366:799-807.

14. Tefferi A, Litzow MR, Pardanani A. Long-term outcome of treatment with ruxolitinib in myelofibrosis. N Engl J Med 2011;365:1455-1457.

15. Tefferi A, Pardanani A. Serious adverse events during ruxolitinib treatment discontinuation in patients with myelofibrosis. Mayo Clin Proc 2011;86:1188-1191.

16. Dai T, Friedman EW, Barta SK. Ruxolitinib withdrawal syndrome leading to tumor lysis. J Clin Oncol 2013;31:e430-432.

17. Verstovsek S, Kantarjian H, Mesa RA, et al. Safety and efficacy of INCB018424, a JAK1 and JAK2 inhibitor, in myelofibrosis. New Engl J Med 2010;363:1117-1127.

18. Czene K, Adami HO, Chang ET. Sex- and kindred-specific familial risk of non-Hodgkin's lymphoma. Cancer Epidemiol Biomarkers Prev 2007;16:2496-2499.

19. Fisher SG, Fisher RI. The epidemiology of non-Hodgkin's lymphoma. Oncogene 2004;23:6524-6534.

20. Bairey $O$, Benjamini $O$, Blickstein D, et al. Non-Hodgkin's lymphoma in patients 80 years of age or older. Ann Oncol 2006;17:928-934.

21. Cuttner J, Wallenstein S, Troy K. Non-Hodgkin's lymphoma in patients 70 years of age or older: factors associated with survival. Leuk Res 2002;26:447-450.

22. Palandri F, Derenzini E, Ottaviani E, et al. Association of essential thrombocythemia and non-Hodgkin lymphoma: a single-centre experience. Leuk Lymphoma 2009;50:481-484 choice questions. Credit cannot be obtained for tests completed on paper. You must be a registered user on NCCN.org. If you are not registered on NCCN.org, click on "New Member? Sign up here" link on the left hand side of the Web site to register. Only one answer is correct for each question. Once you successfully answer all posttest questions you will be able to view and/or print your certificate. Software requirements: Internet.

\section{Posttest Questions}

1. True or False: Essential thrombocythemia is well-known to transform to other myeloid disorders such as leukemia. Additionally, patients are at an increased risk of developing lymphoid malignancies, such as NHL and nonhematologic malignancies.

2. Ruxolitinib therapy results in significant suppression of inflammatory cytokines; abrupt cessation of ruxolitinib is believed to result in cytokine rebound, resulting in withdrawal syndrome. Which of the following is NOT true about ruxolitinib-induced withdrawal syndrome?

a. Gradual cessation of ruxolitinib may prevent this complication.

b. Withdrawal syndrome may manifest as symptom relapse, painful enlargement of spleen, septic shock-like syndrome, disseminated intravascular coagulation, and tumor lysis syndrome.

c. Reinitiation of ruxolitinib and the use of glucocorticoids may be useful in its management.

d. Ruxolitinib-induced withdrawal syndrome is not seen in patients with primary myelofibrosis.

3. True or False: The risk of leukemic transformation is the highest within the first 5 years of diagnosis of essential thrombocythemia. This risk significantly decreases among patients who survive beyond the first decade. 